\title{
Extra-Large Seed Germplasms of Brassica Napus Created through Microspore Culture
}

\author{
Chen $\mathrm{Wei}^{1}$, Zhao Kaiqin ${ }^{1}$, Zhang Guojian ${ }^{2}$, Zhou Picai $^{3}$, Zhao Desheng ${ }^{3}$, Qu Guan ${ }^{4}$, Luo Yangqing ${ }^{1}$, Zu Feng $^{1}$, Li \\ Jingfeng $^{1}$, Dong Yunsong ${ }^{1}$ and Wang Jingqiao ${ }^{1}$ \\ 1. Research Institute of Industrial Crops, Academy of Agricultural Science, Kunming 650205, China \\ 2. Department of Agriculture, Yunnan Vocational and Technical College of Agriculture, Kunming 650031, China \\ 3. Institute of Agricultural Promotion, Academy of Agricultural Science, Chuxiong 675000, China \\ 4. Research Institute of Oil Crops, Academy of Agricultural Science, Yuxi 653100, China
}

\begin{abstract}
Thousand Seed Weight (TSW) is one of the major yield components of rapeseed (Brassica napus L.). Here reports an extra-large seed germplasm GM01 which was obtained through isolated microspore culture. Three-way cross was made: H8 - a Yunnan spring early-maturing rapeseed variety, "Legency" — a Canadian canola variety and "020010"—a semi-winter late-maturing rapeseed variety. One hundred and forty eight doubled hyploid lines were obtained from the $\mathrm{F}_{1}$ plants of three-way cross through isolated microspore culture. Among them, the TSW of GM01 amounted to $8.68 \mathrm{~g}$ and the TSW of 53 lines were above $5.0 \mathrm{~g}$. The TSWs of GM01 were relatively stable among the multi-location field trials from 2007 to 2014 with variation being only $10 \%-15 \%$ among the locations and years. Compared with H8, GM01 had larger flowers, stigmas, siliques and seed diameters, but less branches, siliques per plant and seeds per silique.
\end{abstract}

Key words: Brassica napus, germplasm, seed weight, morphological trait, microspore culture.

\section{Introduction}

Rapeseed (Brassica napus L.) is one of the important oil crops for edible plant oil, other staples, such as rice, maize and wheat, which continuously improve the seed yield is always the major objective for rapeseed breeders. Rapeseed yield per unit area is determined by two main components, that is, the number of seeds per unit area and the seed weight [1, 2]. The number of seeds per unit area is closely related to the number of branches per plant, the number of pods per plant and the number of grains per pod [3-5]. Therefore, high yielding can be obtained by increasing either the number of seeds per plant, 1,000-seed weight (TSW) or the both $[6,7]$. At present, the yield of main cultivated varieties of rapeseed was about 2,500-4,000 kg/hr and TSWs of the varieties were in the range of $3.0 \mathrm{~g}$ to $3.5 \mathrm{~g}$ [1]. According to the

Corresponding author: Wang Jingqiao, master, research field: modern biotechnology breeding. assumption of Berry, P. M. [1], in order to obtain the seed yield of $6,000 \mathrm{~kg} / \mathrm{hr}$, the TSW of cultivar should reach $5.0 \mathrm{~g}$ and for the seed yield of $9,000 \mathrm{~kg} / \mathrm{hr}$, TSW of should be up to $6.10 \mathrm{~g}$. Thus, high TSW should be one of the most important characteristics of excellent variety.

Germplasm resources are the material foundation for crop breeding. Rapeseed (AACC, $2 n=38$ ) is allopolyploid. It originates about 0.12-1.37 million years ago in the Mediterranean region by natural hybridization between Brassica rapa (AA, $2 \mathrm{n}=20$ ) and Brassica oleracea $(\mathrm{CC}, 2 \mathrm{n}=18)[8,9]$, and has a short domestication history of only 400-500 years [10, 11]. Because of its high yield and wide adaptability, rapeseed replaces Brassica rapa and Brassica juncea and becomes the plants worldwide gradually. Comparing to Brassica rapa and Brassica juncea, rapeseed has short evolution history and intensive selection in the traditional breeding process, which resulted in its poor genetic diversity. The poor genetic 
diversity has become the bottleneck of rapeseed breeding and utilization of heterosis [12, 13]. According to the statistics of International Board for Plant Genetic Resources (IBPGR), there were about 43,000 accessions of Brassica germplasm. In these accessions, Brassica rapa, Brassica juncea and rapeseed accounted for $34.09 \%, 27.27 \%$ and $14.32 \%$ of total accessions respectively. In 1996 to 1999, Liu, H. L. [14] collected 871 rapeseed accessions and Brassica juncea germplasms from the western region of China. Among these accessions: the TSWs of the wild germplasm were less than $1.0 \mathrm{~g}$; the average TSWs of cultivated Brassica rapa were 2.34-4.36 g, up to $7.7 \mathrm{~g}$; the average TSWs of cultivated Brassica juncea were 1.66-5.04 g, up to $8.0 \mathrm{~g}$ and the average TSWs of rapeseed were 2.0-4.0 g. Chinese rapeseed germplasms were mainly introduced from European countries. The TSWs of currently cultivated rapeseed varieties ranges from $3.0 \mathrm{~g}$ to $3.5 \mathrm{~g}$, indicating the scarity of large seed rapeseed germplasms compared with Brassica rapa and Brassica juncea. Therefore, it is necessary to create new rapeseed germplasms with good economic traits, especially high TSW and oil content.

Here, creation of an extra-large seed rapeseed germplasm GM01 with the TSW of $8.68 \mathrm{~g}$ was reported. It was selected from the $\mathrm{DH}$ (Double Haploid) lines of a three-way cross: "020010"-a semi-winter late-maturing rapeseed variety, "Legency"- a spring Canadian variety and H8-a spring early-maturity Yunnan local rapeseed variety. Among these DH lines, 53 lines had TSW more than 5 g. In 2010-2011, GM01 was evaluated in four locations of Yunnan, China (Kunming, Chuxiong, Lijiang and Xiaoshao) and two locations of Hubei, China (Wuhan city and Ezhou).

\section{Material and Methods}

\subsection{Experimental Procedures}

In the February, 2002, a cross was made between a semi-winter late-maturing rapeseed germplasm " 020010 "
(TSW of $4.6 \mathrm{~g}$ ) and a spring Canadian variety "Legency" (TSW of $3.8 \mathrm{~g}$ ). The $F_{1}$ seeds were harvested and planted in the June of 2002 and a cross was made between F1 of 020010/Legency and a Yunnan spring early-maturing rapeseed variety $\mathrm{H} 8$ (TSW of $3.6 \mathrm{~g}$ ) (all the materials were deposited in the genetic stock of the Yunnan Academy of Agricultural Sciences). The $F_{1}$ of the cross $020010 /$ Legency/H8 was planted in the October, 2002 and the buds of the $F_{1}$ plants were used to isolate microspores for culture. One hundred and forty eight DH lines were obtained and their TSWs were evaluated in 2004 and 2005. Among these DH lines, 69 lines had TSW of 4.0-5.0 g and 53 lines had TSW more than $5 \mathrm{~g}$. Five lines with TSW higher than $7.0 \mathrm{~g}$ and $\mathrm{H} 8$ were evaluated in four locations mentioned above in the winter season of 2010-2014. The trials were set up in a Complete Randomized Block Design (RCBD) with three replications. Seeds had been handed planted in $2 \times 2 \mathrm{~m}$ plots. Each plot contained 5 rows with $40 \mathrm{~cm}$ space between the rows and 16 plants per row with $15 \mathrm{~cm}$ space.

\subsection{Crop Assessment}

The morphological characteristics of GM01 and H8 were measured in the Kunming winter trial of 2010-2011. The length and width of petals, the length of stigmas and pistils, the diameter of pedicel, ovule number and the fresh weight of flower buds and ovaries were averaged from 50 fresh flower bud or flowers from the main stem at the beginning of flowering.

Agronomic traits including plant height, pods per plant, number of seeds per pod, TSW and yield per plant were averaged from ten randomly selected plants at maturity. The length and width of the silique were averaged from 50 siliques. The seed diameter was averaged from randomly selected 100 seeds from the 50 siliques. Seeds were air dried and were used in quality analysis with near-infrared FOSS analyzer.

Data were subjected to analysis of variance (ANOVA) or simple $t$-test using SPSS software. 


\section{Results}

\subsection{The Creation of GM01}

In 2002, the three-way cross "020010/legency//H8" was obtained. In 2003, 148 DH plants were obtained from the isolated microspore culture of $F_{1}$ plants of “020010/legency//H8". These DH lines segregated in their TSW in the Kunming trail (Fig. 1). Among those DH lines, 53 lines had TSW over $5.0 \mathrm{~g}$ and the biggest TSW amounted to $8.68 \mathrm{~g}$. According to TSW of the 2003 trial, $12 \mathrm{DH}$ lines of high $\mathrm{TSW}$ (> $5.0 \mathrm{~g}$ ) were selected for the winter trials of 2004 and 2005. The TSW of these DH lines were relatively stable between two years with variation of 10\%-15\% (Fig. 2).

Based on the field experiment of high TSW of GM01 line for multi-years and locations in different ecological environments, its TSW ranged from $6.2 \mathrm{~g}$ to $7.6 \mathrm{~g}$ and was significantly higher than the control H8, despite the impact of the various ecological environment of the planting years (Table 1).

\subsection{Morphological Characteristics of GM01}

\subsubsection{Corolla Characteristics of GM01}

Four petals of H8 and GM01 were all arranged in a typical cross figure. The petals of $\mathrm{H} 8$ were flat on the top whereas, those of GM01 were folded on the top. The four petals of H8 were largely separated from each other, whereas, those of GM01 were overlapped between two adjacent petals (Fig. 3). The lengths of the petals of GM01 and H8 were similar, but the widths of the petals of GM01 were about $30 \%$ broader than those of H8 (Table 2). The pedicel of GM01 was in the same length with that of $\mathrm{H} 8$, but it was about $78 \%$ thicker than that of H8. The lengths of flower bud were the same between GM01 and H8, and the diameter and fresh weight of flower buds of GM01 were higher than those of H8. The length of the ovary was the same between GM01 and H8 and they also

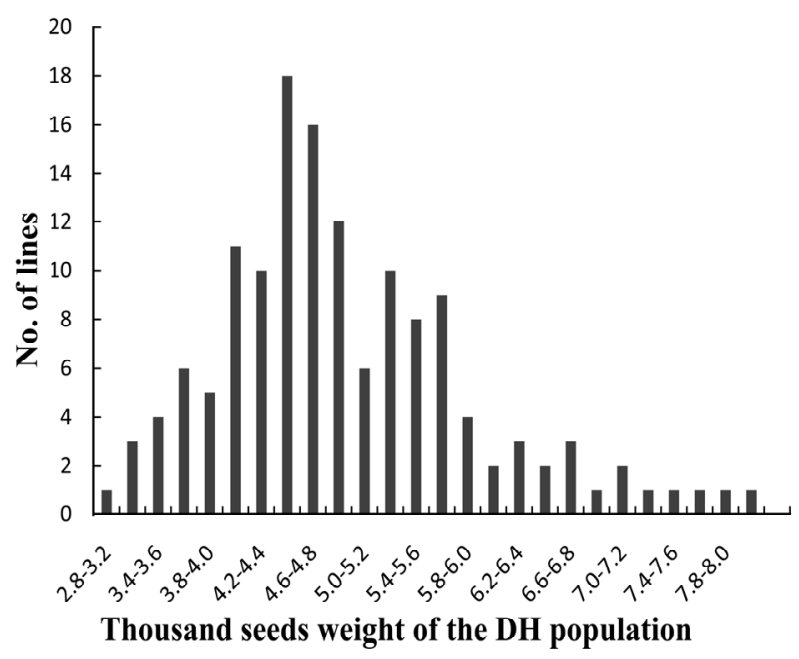

Fig. 1 TSW of $148 \mathrm{DH}$ lines in the Kunming trial of 2003.

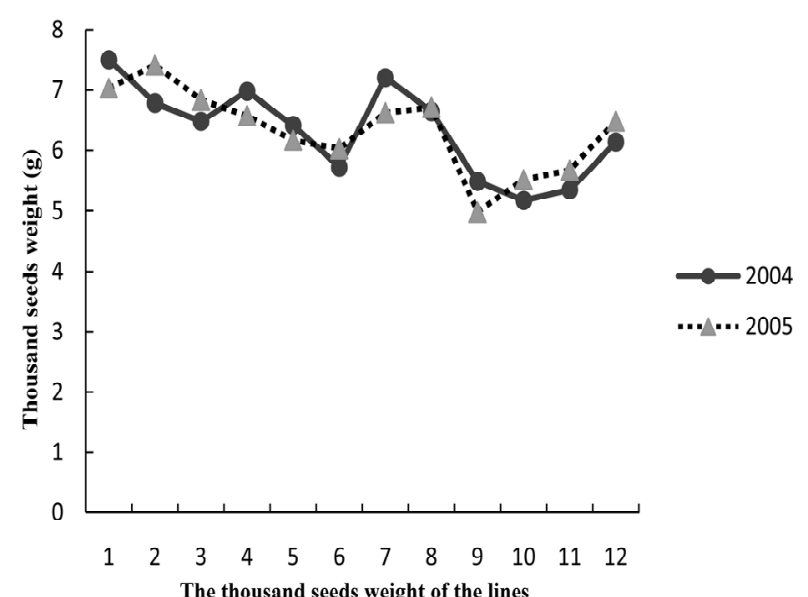

Fig. 2 TSW of 12 big seed DH lines in the Kunming winter trials of 2004 and 2005.

Table 1 TSW of GM01 in the trials of six locations in 2010-2014.

\begin{tabular}{lllllll}
\hline \multirow{2}{*}{ Location } & \multirow{2}{*}{ Latitude } & \multirow{2}{*}{ Altitude $(\mathrm{m})$} & \multicolumn{4}{c}{$\mathrm{TSW}(\mathrm{g})$} \\
\cline { 4 - 6 } & & & 2010 & 2011 & 2013 & 2014 \\
\hline Kunming & $\mathrm{N} 25^{\circ} \mathrm{E} 102^{\circ}$ & 1,910 & $7.15 \pm 0.45^{* *}$ & $7.63 \pm 0.51^{* *}$ & $7.02 \pm 0.55^{* *}$ & $7.09 \pm 0.22^{* *}$ \\
Xiaoshao & $\mathrm{N} 25^{\circ} \mathrm{E} 103^{\circ}$ & 1,960 & $6.90 \pm 0.57^{* *}$ & $7.33 \pm 0.77^{* *}$ & $7.22 \pm 0.41^{* *}$ & $6.89 \pm 0.43^{* *}$ \\
Cuxiong & $\mathrm{N} 25^{\circ} \mathrm{E} 102^{\circ}$ & 1,870 & $6.77 \pm 0.37^{* *}$ & $6.92 \pm 0.57^{* *}$ & $7.01 \pm 0.61^{* *}$ & $7.13 \pm 0.31^{* *}$ \\
Lijiang & $\mathrm{N} 26^{\circ} \mathrm{E} 110^{\circ}$ & 2,400 & $6.88 \pm 0.41^{* *}$ & $7.12 \pm 0.67^{* *}$ & $6.79 \pm 0.34^{* *}$ & - \\
Wuhan & $\mathrm{N} 30^{\circ} \mathrm{E} 112^{\circ}$ & 27 & $7.03 \pm 0.38^{* *}$ & $7.11 \pm 0.56^{* *}$ & $7.21 \pm 0.45^{* *}$ & $6.27 \pm 0.47^{* *}$ \\
Ezhou & $\mathrm{N} 30^{\circ} \mathrm{E} 114^{\circ}$ & 20 & $7.03 \pm 0.33^{* *}$ & $7.07 \pm 0.25^{* *}$ & $7.21 \pm 0.35^{* *}$ & - \\
\hline
\end{tabular}

$*$ and $* *$ refer to the significance of differences at the 0.05 and 0.01 level respectively. 

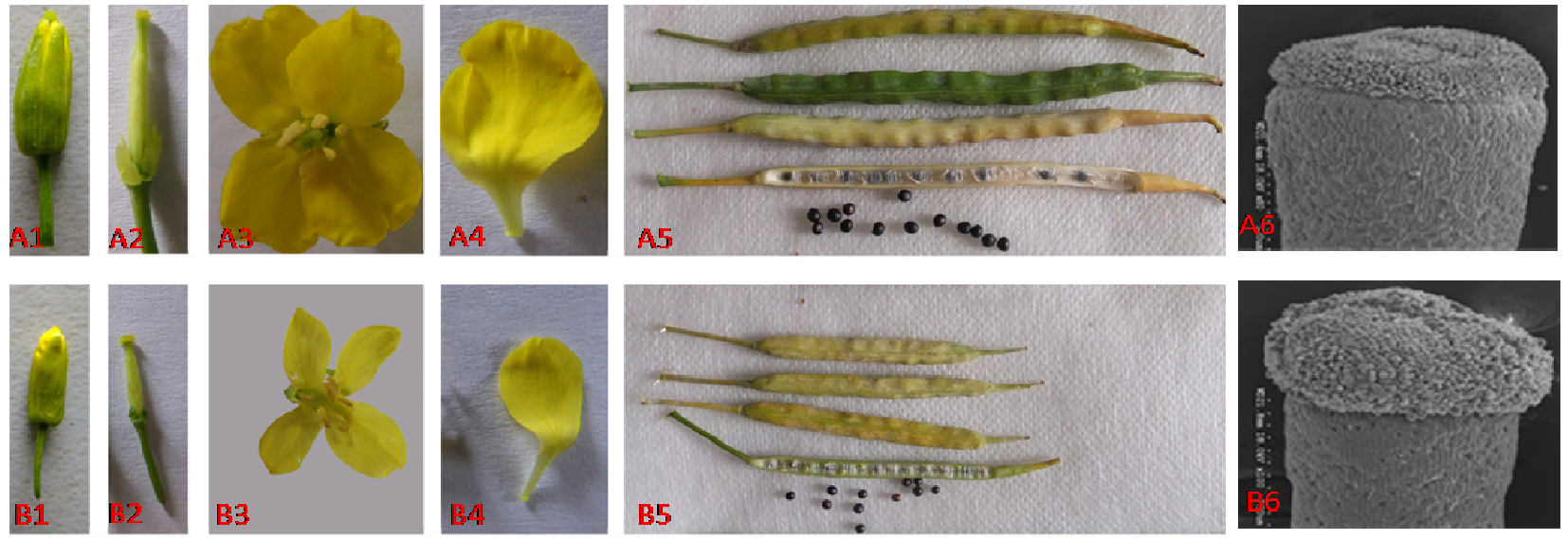

Fig. 3 The flowers, siliques, stigma and seeds of GM01 and H8 (A: extra-large seed material "GM01"; A1: flower bud; A2: ovary; A3: flower; A4: petal; A5: pods; A6: stigma under scanning electron microscope $(\times 80)$; B: average seed material "H8"; B1: flower bud; B2: ovary; B3: flower; B4: petal; B5: pods and B6: stigma under scanning electron microscope $(\times$ 100)).

Table 2 The flower organ characteristics of GM01 and H8 in Kunming trial of 2010.

\begin{tabular}{lllll}
\hline Flower organ & Measurements & GM01 & H8 & Lengency \\
\hline \multirow{2}{*}{ Petal } & Length $(\mathrm{cm})$ & $1.25 \pm 0.22$ & $1.24 \pm 0.09$ & $1.33 \pm 0.11$ \\
Pedicel & Width $(\mathrm{cm})$ & $0.96 \pm 0.02^{* *}$ & $0.66 \pm 0.07$ & $0.57 \pm 0.04$ \\
& Diameter $(\mathrm{mm})$ & $0.82 \pm 0.08^{* *}$ & $0.46 \pm 0.07$ & $0.41 \pm 0.07$ \\
Flower bud & Length $(\mathrm{cm})$ & $7.51 \pm 0.17$ & $7.13 \pm 0.38$ & $0.69 \pm 0.12$ \\
& Diameter $(\mathrm{cm})$ & $4.07 \pm 0.17^{* *}$ & $3.4 \pm 0.12$ & $3.3 \pm 0.17$ \\
& Fresh weight (g) & $49.05 \pm 2.32^{* *}$ & $35.14 \pm 2.64$ & $32.33 \pm 1.66$ \\
Ovary & Length $(\mathrm{cm})$ & $0.82 \pm 0.02$ & $0.79 \pm 0.05$ & $0.77 \pm 0.07$ \\
& Fresh weight (g) & $7.73 \pm 0.59^{* *}$ & $5.53 \pm 0.53$ & $5.69 \pm 0.47$ \\
Stamen & Ovule & $30.33 \pm 1.97$ & $31.01 \pm 1.33$ & $31.41 \pm 1.13$ \\
Pistil & Length $(\mathrm{cm})$ & $0.65 \pm 0.065$ & $0.68 \pm 0.042$ & $0.62 \pm 0.033$ \\
Silique & Length $(\mathrm{cm})$ & $0.71 \pm 0.021^{* *}$ & $0.67 \pm 0.013$ & $0.64 \pm 0.021$ \\
& Length $(\mathrm{cm})$ & $12.11 \pm 0.86^{* *}$ & $6.33 \pm 0.77$ & $6.71 \pm 0.83$ \\
Seed & Width $(\mathrm{mm})$ & $5.98 \pm 0.49^{* *}$ & $4.87 \pm 0.36$ & $4.41 \pm 0.25$ \\
& Diameter $(\mathrm{mm})$ & $2.62 \pm 0.74^{* *}$ & $1.42 \pm 0.44$ & $1.29 \pm 0.39$ \\
& Fresh weight (g/1,000 seeds) & $7.20 \pm 1.13 * *$ & $3.47 \pm 0.87$ & $3.79 \pm 0.77$ \\
\hline
\end{tabular}

$*$ and $* *$ refer to the significance of differences at the 0.05 and 0.01 level respectively.

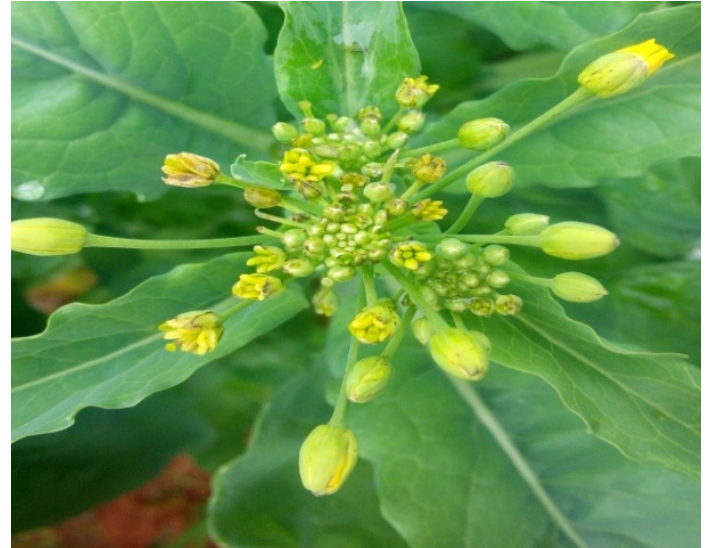

Fig. 4 Stigma exsertion phenomenon of GM01. bared the similar number of ovules, but the fresh weight of GM01 ovary was about $40 \%$ higher than that of $\mathrm{H} 8$ (Table 2).

\subsubsection{The Pistil and Stigma of GM01}

The pistil of GM01 grew quicker than its stamens and the stigma was longer than stamens, thus, the stigma projected from the stamens. Then, about 30\% GM01 plants had stigma exsertion phenomenon, especially in summer (Fig. 4). Whereas, the pistil of H8 was equal to or less than its stamens and the stigma was parallel or below the anthers. Comparing 
the tip of stigma of GM01 and H8, H8 had more mastoid cells at the tip of stigma than GM01, therefore, the mastoid cells appeared to be hemispherical hat over the style of $\mathrm{H} 8$ while mastoid cells only covered the top of the style of GM01 (Fig. 3A6).

\subsubsection{The Siliques and Seeds of GM01}

GM01 had characteristics of long silique, thick silique skin and big seed. The silique length, width and seed diameter of GM01 were significantly greater than those of H8 (Table 3, Fig. 3A5). The fresh weight and dry weight of GM01 siliques were three times of those of H8. The dry weight of seed and embryo were 2.5 times of those of $\mathrm{H} 8$ (Table 3 ).

\subsection{The Main Agronomic Traits of Big Seed Germplasms}

Five big seed DH lines including GM01, GM02, GM03, GM04 and GM05 were selected for two year. The days to maturity of these $\mathrm{DH}$ lines were all more than 185 days, much longer than 182 days for $\mathrm{H} 8$. Comparing to $\mathrm{H} 8$, these five big seed $\mathrm{DH}$ lines had mainly primary branches, but no or few secondary branches. They had less siliques per plant and seeds per silique. However, because of much higher TSWs of these five DH lines, their yield per plant was significantly higher than that of H8 (Table 4).

\subsection{Quality Traits of Five DH Lines and H8}

The seed oil contents of GM01, GM02, GM03, GM04 and GM05 ranged from $38.18 \%$ to $42.13 \%$ with little advantage over that of H8. Their seed protein contents ranged from $23.95 \%$ to $25.94 \%$, which were higher about $0.3 \%-2 \%$ than that of $\mathrm{H} 8$. However, the glucosinolate and erucic acid contents of these $\mathrm{DH}$ lines were significantly higher than that of H8 (Table 5).

\section{Discussion}

TSW is a complex quantitative traits controlled by the main genes, plus multiple minor genes, and easily affected by environment $[15,16]$. The probability of obtaining big seed lines through conventional hybridization breeding is only $1 / 1,000$ to $1 / 30,000$, thus, it is difficult to obtain heritable large seed materials.

Isolated microspore culture is a cell (haploid) culture-based approach for producing completely homozygous DH plants from immature pollen grains

Table 4 Main agronomic traits of new germplasm.

\begin{tabular}{lllllll}
\hline \multirow{2}{*}{ Traits } & \multicolumn{5}{c}{ Lines } \\
\cline { 2 - 7 } & GM01 & GM02 & GM03 & GM04 & GM05 & H8 (CK) \\
\hline Days of growth duration (days) & 201 & 186 & 191 & 185 & 195 & 182 \\
Height of plant (cm) & $151.7 \pm 5.17$ & $148.8 \pm 4.96$ & $150.5 \pm 2.17$ & $152.7 \pm 2.41$ & $163.3 \pm 3.14$ & $157.3 \pm 1.12$ \\
Diameter of main stem (mm) & $19.55 \pm 0.34$ & $17.66 \pm 1.09$ & $16.24 \pm 0.89$ & $16.21 \pm 0.66$ & $16.51 \pm 1.02$ & $15.31 \pm 0.52$ \\
No. of branch & $8.7 \pm 1.31$ & $10.9 \pm 0.96$ & $7.4 \pm 1.12$ & $8.3 \pm 0.33$ & $11.1 \pm 0.73$ & $11.8 \pm 0.24$ \\
Pods per plant & $275.3 \pm 15.42$ & $262.6 \pm 20.69$ & $278.7 \pm 11.13$ & $206.2 \pm 26.13$ & $295.4 \pm 11.35$ & $357.4 \pm 10.09$ \\
Seeds per pod & $17.2 \pm 0.33$ & $18.2 \pm 0.56$ & $15.9 \pm 0.27$ & $19.4 \pm 0.53$ & $17.8 \pm 1.02$ & $21.6 \pm 0.24$ \\
1,000 seed weight $(\mathrm{g})$ & $7.22 \pm 1.17$ & $7.12 \pm 1.31$ & $7.03 \pm 0.59$ & $6.78 \pm 0.6$ & $6.33 \pm 0.65$ & $3.37 \pm 0.42$ \\
Diameter of seed (mm) & $3.24 \pm 0.06$ & $3.21 \pm 0.13$ & $3.17 \pm 0.07$ & $3.07 \pm 0.35$ & $3.23 \pm 0.14$ & $1.87 \pm 0.03$ \\
\hline
\end{tabular}

Table 5 The component analysis of seeds of DH lines and $\mathrm{H8}$.

\begin{tabular}{lllll}
\hline Lines & Oil content $(\%)$ & Protein content $(\%)$ & Glucosinolate content $(\mu \mathrm{mol})$ & Erucic acid $(\%)$ \\
\hline GM01 & $38.18 \pm 0.58$ & $25.8 \pm 0.76$ & $139.82 \pm 15.51$ & $33.89 \pm 3.93$ \\
GM02 & $38.31 \pm 0.71$ & $25.94 \pm 0.83$ & $132.29 \pm 13.27$ & $31.24 \pm 5.86$ \\
GM03 & $41.66 \pm 0.73$ & $23.73 \pm 1.02$ & $93.25 \pm 11.13$ & $25.11 \pm 2.14$ \\
GM04 & $42.13 \pm 0.51$ & $23.95 \pm 0.77$ & $89.52 \pm 5.93$ & $24.17 \pm 5.71$ \\
GM05 & $38.76 \pm 0.81$ & $25.1 \pm 0.93$ & $96.06 \pm 7.53$ & $23.31 \pm 4.05$ \\
H8 (CK) & $39.55 \pm 0.73$ & $23.48 \pm 0.23$ & $17.62 \pm 1.06$ & $1.11 \pm 0.57$ \\
\hline
\end{tabular}


in a single generation $[17,18]$. This technology is widely used in rapeseed breeding and its genetic analysis $[19,20]$ and has been adopted in many laboratories [21-24].

In this study, one out of $148 \mathrm{DH}$ plants had TSW over 7 grams and other two DH plants had average TSW more than 7 grams in multi-location trials in 2004 and 2005 (Fig. 2). The probability of obtaining plants with TSW over $7.0 \mathrm{~g}$ was from $1 / 148$ to $3 / 148$, which was significantly higher than that of the conventional breeding.

The newly created DH lines (GM01, GM02, GM03, GM04 and GM05) not only produced big seed with TSW of $7.0 \mathrm{~g}$ in high altitude regions such as Kunming, Chuxiong and Lijiang of Yunan province, but also in low altitude plain of Wuhan and Ezhou of Hubei province. This indicates that there are major genes controlling the big seed characteristic in these DH lines.

\section{Acknowledgement}

NSFC (National Science Foundation of China) 30860147 and 31260338, Yunnan natural science foundation of China 2011FB117, “863" High Technology $R$ and $D$ Project of China (2011AA10A104), Scientific Observing and Station of Potato and Rapeseed in Yunnan-Guizhou Plateau, Ministry of Agriculture, Kunming, China.

\section{References}

[1] Berry, P. M., and Spink, J. H. 2006. "A Physiological Analysis of Oilseed Rape Yield: Past and Future.” J. Agri. Sci. 144: 381-392.

[2] Shi, J. Q., Li, R. Y., Qiu, D., Jiang, C. C., Long, Y., and Morgan, C. et al. 2009. "Unraveling the Complex Trait of Crop Yield with Quantitative Trait Loci Mapping in Brassica Napus." Genetics 182: 851-861.

[3] Chen, W., Zhang, Y., Liu, X. P., Chen, B. Y., Tu, J. X., and $\mathrm{Fu}, \mathrm{T}$. D. 2007. "Detection of QTL for Six Yield-Related Traits in Oilseed Rape (Brassica Napus L.) Using DH and Immortalized $\mathrm{F}_{2}$ Populations.” Theor. Appl. Genet. 115: 849-858.

[4] Li, Y. Y., Shen, J. X., Wang, T. H., Chen, Q. F., Zhang, X. G., and Fu, T. D. et al. 2007. "QTL Analysis of
Yield-Related Traits and Their Association with Functional Markers in Brassica Napus L.." Australian Journal of Agricultural Research 58: 759-766.

[5] Fan, C. C., Cai, G. Q., Qin, J., Li, Q. Y., Yang, M. G., and $\mathrm{Wu}$, J. Z. et al. 2010. "Mapping of Quantitative Trait Loci and Development of Allele-Specific Markers for Seed Weight in Brassica Napus." Theor. Appl. Genet. 121: 1289-1301.

[6] Abrahae, E., Klima, M., Vyvadilova, M., and Bechyne, M. 2008. "Assessment of Some Agronomic and Seed Quality Traits in Brassica Carinata Landrace Genotypes, Doubled Haploid Lines and Hybrids." Agriculture Tropica Subtropica 41 (4): 150-159.

[7] Hashemi, A. S., Ghorban, A. N., Nadali, B. J., and Omid, G. C. 2010. "Genetic Evaluation of Yield and yield Component at Advanced Generation in Rapeseed (Brassica Napus L.)." Africa Journal of Agricultural Research 5 (15): 1958-1964.

[8] Nagaharu, U. 1935. "Genome Analysis in Brassica with Special Reference to the Experimental Formation of $B$. Napus and Peculiar Mode of Fertilization." Japanese Journal of Botany 7: 389-452.

[9] Cheung, F., Trick, M., and Drou, N. 2009. "Comparative Analysis between Homeoeologous Genome Segment of Brassica Napus and Its Progenitor Species Reveals Extensive Sequence-Level Divergence." The Plant Cell 21 (7): 1912-1928.

[10] Gomez-Campo, C., and Parkash, S. 1999. "Origin and Domestication." Biology of Brassica Coenospecies 4: 33-58.

[11] Parkash, S., Wu, X. M., and Bhat, S. R. 2011. "History Evolution and Domestication of Brassica Crops." Plant Breeding Reviews 35: 19-84.

[12] Becker, H. C., Engqvist, G. M., and Karlesson, B. 1995. "Comparison of Rapeseed Cultivars and Resynthesized Lines Based on Allozyme and RELP Marks." Theoretical and Applied Genetics 91 (1): 62-67.

[13] Malek, M. A., Ismail, M. R., Rafii, M. Y., and Rahman, M. 2012. "Synthetic Brassica Napus L.: Development and Studies on Morphological Characters, Yield Attributes and Yield." The Scientific World Journal 2012: 1-6.

[14] Liu, H. L. 2000. Rapeseed Breeding and Genetics (in Chinese). Beijing: China Agricultural University Press.

[15] Qi, C. K., Gai, J. Y., Fu, S. Z., Pu, H. M., Zhang, J. F., and Chen, X. J. et al. 2004. "Analysis of Genetic System of 1,000 Seed Weight in Brassica Napus L." Acta. Agron. Sin. 30: 1274-1277.

[16] Yang, P., Shu, C., Chen, L., Wu, J., and Liu, K. 2012. "Identification of a Major QTL for Silique Length and Seed Weight in Oilseed Rape (Brassica Napus L.)." Theor. Appl. Genet. 125: 285-296. 
[17] Iva, S., Martina, V., Miroslav, K., Ivana, M., and Miroslav, G. 2006. "Efficiency of Microspore Culture for Doubled Haploid Production in the Breeding Project-Czech Winter Rape." Czech J. Genet. and Plant Breed 42 (2): 58-71.

[18] Takahashi, Y., Yokoi S., and Takahata, Y. 2011. "Improvement of Microspore Culture Method for Multiple Sample in Brassica." Breeding Science 61: 96-98.

[19] Alison, M. R., and Ferrie-Christian, M. 2011. "Haploids and Doubled Haploids in Brassica Spp. for Genetic and Genomic Research." Plant Cell Tiss. Organ Cukt. 104: 375-386.

[20] Rajcan, I., Kasha, K. J., Kott, L. S., and Beversodrf, W. D. 2002. "Evaluation of Cytoplasmic Effects on Agronomic and Seed Quality Traits in Two Doubled Haploid Populations of Brassica Napus L.." Euphytica 123: 401-409.
[21] Francisco, B., Juan, F. E., Mercedes, V., and Martín, A. 2002. "Modification of Glucosinolate and Erucic Acid Contents in Doubled Haploid Lines of Brassica Carinata by UV Treatment of Isolated Microspores." Euphyica 129: $1-6$.

[22] Vinitha, C., and Neal, Stert, C. 2004. "Invited Review: Brassica Biotechnology: Progress in Cellular and Molecular Biology." In Vitro Dev. Biol-Plant 40: 542-551.

[23] Jiha, K., and Soo-Seong, L. 2012. "Identification of Monogenic Dominant Male Sterility and Its Suppressor Gene from Induced Mutation Using a Broccoli (Brassica Oleracea Var. Italica) Microspore Culture." Hort. Environ. Biotechnol 53 (3): 237-241.

[24] Deepak, P., Kadambari, G., and Abha, A. 2012. "Harnessing Mutant Donor Plants for Microspore Culture in Indian Mustard (Brassica Juncea (L.) Czern and Coss)." Euphytica 184: 207-222. 